\title{
Clinicopathologic Evaluation of Lesions associated with Tobacco Usage
}

\author{
${ }^{1}$ Yalamanchili Samatha, ${ }^{2}$ Avula Jogendra Sai Sankar, ${ }^{3} \mathrm{KS}$ Ganapathy, ${ }^{4}$ Kandula Srinivas \\ ${ }^{5}$ Dasari Ankineedu, ${ }^{6}$ Alluri Leela Subhashini Choudary
}

\begin{abstract}
Introduction: Tobacco usage in different forms is the single most common etiological factor responsible for oral cancers. The aim of the present study was to record various mucosal lesions associated with tobacco usage and to ascertain the prevalence of dysplasia in them by histopathological evaluation and to compare the extent of dysplastic features seen among patients associated with a habit of smoked and smokeless form of tobacco.
\end{abstract}

Materials and methods: Seventy-six patients with the clinical diagnosis of tobacco related lesions (Leukoplakia, Erythroplakia, Nicotina stomatitis, Tobacco pouch keratosis) were selected. A detailed description of the clinical presentation of the lesion was noted and the patients were subjected to incisional biopsy followed by a histopathological evaluation.

Results: Showed dysplastic changes in 50 cases $(65.8 \%)$ ranging from mild dysplasia in 27 cases $(35.5 \%)$, moderate dysplasia in 17 cases $(22.4 \%)$ and 6 cases $(7.9 \%)$ showed severe dysplasia. Moderate to severe dysplasia was 1.83 times more likely to occur with smokeless tobacco usage when compared to smoked form. Data obtained from this study reveals that patients with a duration of tobacco usage more than 10 years were 2.17 times more likely to have moderate to severe dysplasia $(p=0.154)$.

Conclusion: Thus, the study highlights the role of oral physicians in detecting oral mucosal lesions and screening high-risk

\footnotetext{
${ }^{1}$ Associate Professor, ${ }^{2}$ Professor and Head, ${ }^{3}$ Former Principal and Head, ${ }^{4}$ Professor, ${ }^{5}$ Resident, ${ }^{6}$ Private Practitioner

${ }^{1}$ Department of Oral Medicine and Radiology, Sibar Institute of Dental Sciences, Guntur, Andhra Pradesh, India

${ }^{2}$ Department of Pedodontics and Preventive Dentistry, Sibar Institute of Dental Sciences, Guntur, Andhra Pradesh, India

${ }^{3}$ Department of Oral Medicine and Radiology, The Oxford Dental College and Research Centre, Bengaluru, Karnataka India

${ }^{4}$ Department of Oral Medicine and Radiology, ACES Maruthi Dental College, Bengaluru, Karnataka, India

${ }^{5}$ Department of Oral Medicine, Sibar Institute of Dental Sciences, Guntur, Andhra Pradesh, India

${ }^{6}$ Guntur, Andhra Pradesh, India
}

Corresponding Author: Yalamanchili Samatha, Associate Professor, Department of Oral Medicine and Radiology, Sibar Institute of Dental Sciences, Guntur, Andhra Pradesh, India e-mail: saisamata@gmail.com patients on a regular basis and also reaffirms the importance of public education, stressing the risk factors for oral cancers.

Keywords: Tobacco, Premalignant, Dysplasia.

How to cite this article: Samatha Y, Sankar AJS, Ganapathy KS, Srinivas K, Ankineedu D, Choudary ALS. Clinicopathologic Evaluation of Lesions associated with Tobacco Usage. J Contemp Dent Pract 2014;15(4):466-472.

Source of support: Nil

Conflict of interest: None

\section{INTRODUCTION}

'From a short pleasure can come a long repentance'.

Tobacco has been variously hailed as a gift from the gods, a miraculous cure-all of life's physical ills, a solace to the lonely soldier or sailor, a filthy habit, a corrupting addiction and the greatest disease producing products known to man. ${ }^{1}$ The open fact is 'Tobacco kills'; killing more than 4 million people each year. It was estimated that 4.9 million people died of tobacco related illness in the year 2000 and by 2020 that figure will rise to 10 million deaths per year, $70 \%$ of which will be in the developing countries. The mouth is among the principal sites in which pathologic conditions have been associated with the smoking habit. A variety of oral mucosal changes attributable to the use of tobacco are observed in approximately $50 \%$ of tobacco users. At the clinical level these mucosal changes may include alteration in color, texture and contour; at the histological level inflammation, vacuolization, epithelial atrophy, acanthosis, hyperorthokeratosis/hyperparakeratosis. These changes include leukoplakia, erythroplakia, nicotina stomatitis, smokeless tobacco keratosis, etc. ${ }^{2}$ In some individuals these lesions may progress to cancer.

Worldwide oral cancer ranges from the 3rd most common cause of death in developing countries to 8th most common cause in developing countries and there is evidence that the incidence and mortality are increasing. ${ }^{3}$ The living cell is a seething mass of activity, which reflects dysfunction when affected by disease. In 1978, the WHO stated that the appearance of oral cancer is preceded by some other lesions in the epithelium, which may show cellular changes that point toward the possible subsequent development of malignancy. The individual cellular changes are referred to 
as atypia and the general disturbance in the epithelium is designated as dysplasia. ${ }^{4}$

Oral epithelial dysplasia is defined as a lesion in which a part of the thickness of the epithelium is replaced by cells showing varying degrees of cellular atypia and maturational disturbances (Pindborg et al 1977). Oral epithelial dysplasia is the histological marker of premalignancy and as such it is predictive of an increased rate of development of squamous cell carcinoma. ${ }^{5}$ The key to better quality and time-span of survival will be, when the disease is detected at a premalignant stage. Thus, the aim of this study was to record the mucosal lesions associated with tobacco related habits and to assess their histopathological status.

\section{METHODOLOGY}

Seventy six patients with the clinical diagnosis of tobacco related lesions (Leukoplakia, Erythroplakia, Nicotina stomatitis, Tobacco pouch keratosis) were selected from the out patients attending the department of Oral medicine and Radiology over a period of one and a half year. Institutional ethical committee approval and an informed written consent from the parents were attained before taking up the study. The selected patients were questioned regarding their tobacco related habits which included the type, frequency and duration of tobacco usage. This was followed by recording case history and a detailed description of the clinical presentation of the lesion. All mucosal lesions were photographed in color using a Nikon camera with $80 \mathrm{~mm}$ macro lens following which an incisinal biopsy was made and the specimens were sent for histopathological evaluation.

The histopathologic findings in each case were recorded under the following categories: (1) various combinations of hyperorthokeratosis, hyperparakeratosis and acanthosis without evidence of dysplasia (2) any combination with mild dysplasia (3) any combination with moderate dysplasia and/or (4) any combination with severe dysplasia/carcinoma in situ. The dysplastic changes were graded for each case as mild, moderate, severe according to the WHO collaborating reference center for oral precancerous lesions, $1978 .^{5}$ Two pathologists evaluated lesions individually (blindfolded of the other person's diagnosis) and when their diagnosis was not in complete agreement, the one with a diagnosis of a greater grade of dysplasia was considered. In the first part of the study, patients with tobacco lesions were categorized according to age, sex, habits and their histopathological status. In the second part, the epithelial dysplasia was correlated with the duration and frequency of tobacco usage and with the type of lesion present clinically.

\section{RESULTS}

The present study was done to record various mucosal lesions associated with tobacco usage in relation to age, sex, duration and frequency of habit and to evaluate their histopathologic findings. In the total sample size of seventy six patients, 59 (77.63\%) were males and 17 (22.37\%) were females with a mean age of 44.05 years (SD 11.85). Smoking associated lesions were seen more in males (96\%) than in females (4\%). Smokeless tobacco keratosis was 24.11 times significantly more in females compared to males with $\mathrm{p}<$ 0.001 .

In the total sample of 76 lesions, $28(36.8 \%)$ were homogenous leukoplakia (Figs 1A and B), 9 (11.8\%) were speckled leukoplakia (Figs 2A and B), one (1.3\%) was erythroplakia, $9(11.8 \%)$ were smoker's palate (Figs $3 \mathrm{~A}$ and $\mathrm{B})$ and $29(38.1 \%)$ were smokeless tobacco keratosis (Figs 4A and B). Of the 76 lesions, 40 (52.6\%) were located on the buccal mucosa and $16(21.1 \%)$ on the commisures. The other sites affected in the decreasing order of frequency were palate $(15.8 \%)$, labial mucosa $(6.6 \%)$ and gingiva, alveolar ridge and tongue with a percentage of $1.3 \%$ each.

Smokeless tobacco keratosis showed the highest occurrence in the buccal mucosa (25 out of 29 cases) which shows

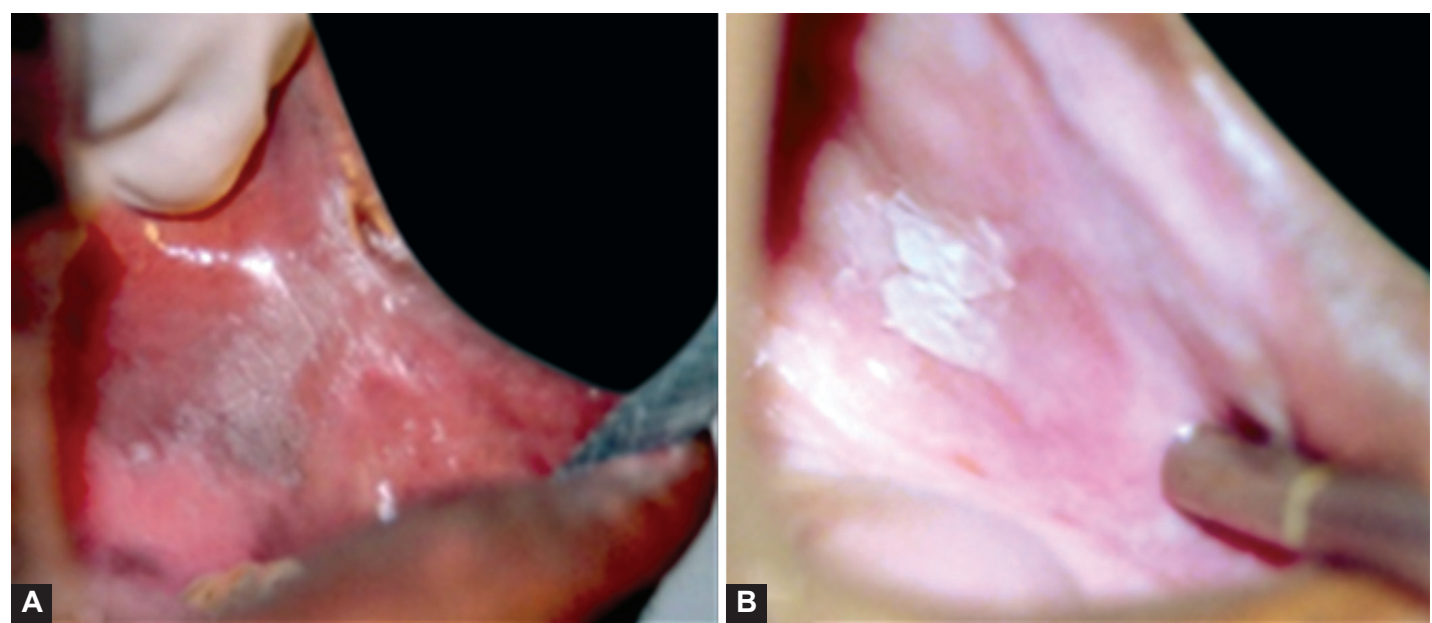

Figs $1 \mathrm{~A}$ and $\mathrm{B}$ : Homogenous leukoplakia in the: $(\mathrm{A})$ left commissural area and $(\mathrm{B})$ in the left buccal mucosa 


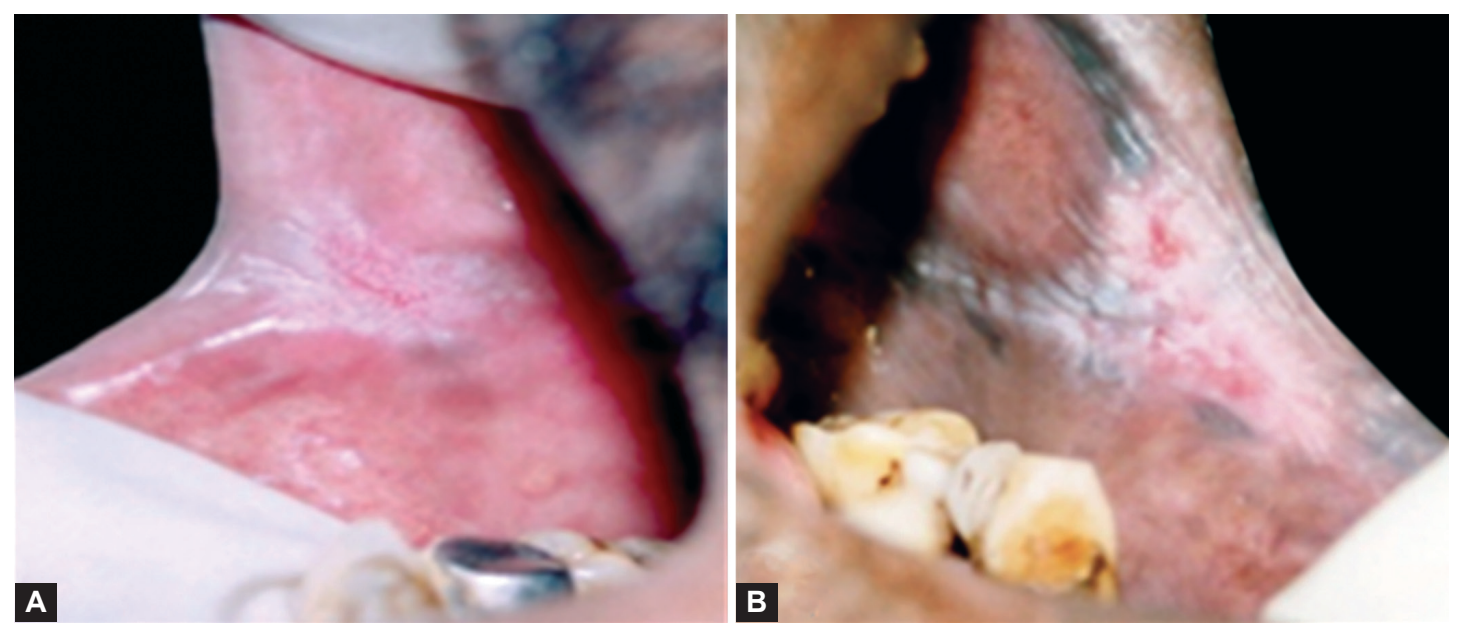

Figs 2A and B: Speckled leukoplakia in the: $(A)$ right and (B) left commissural area
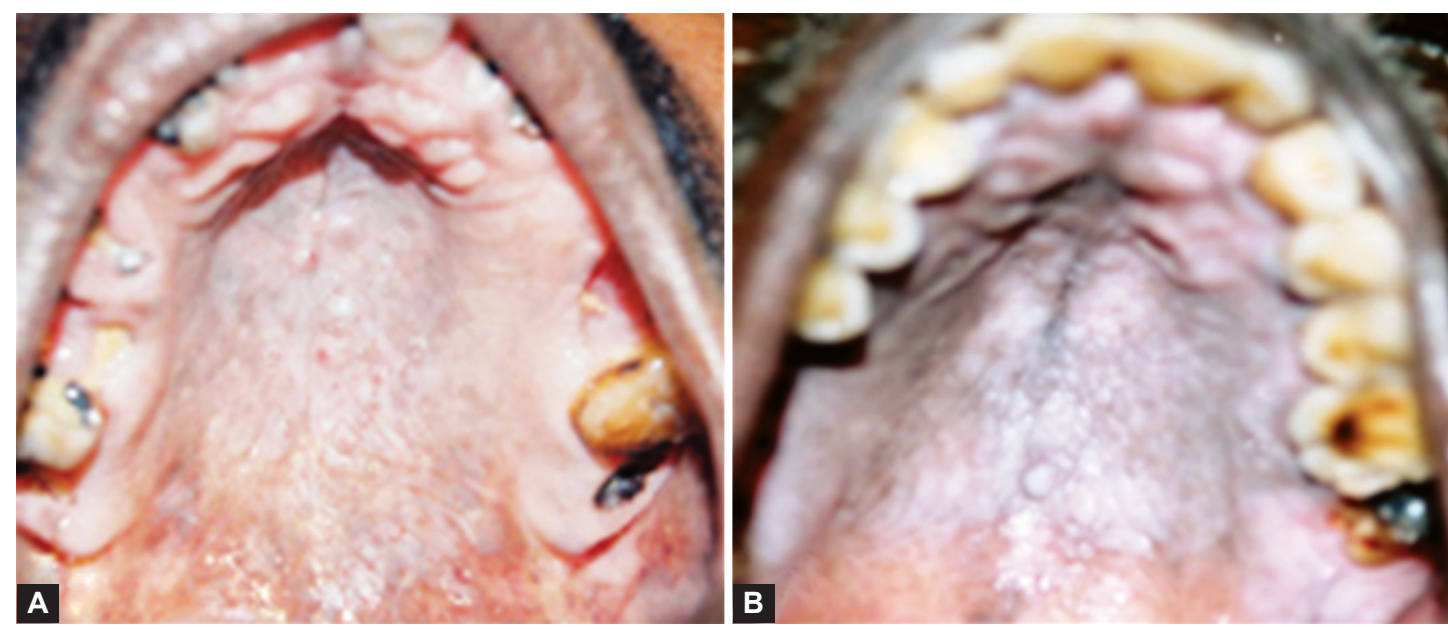

Figs $3 A$ and $B$ : (A) Nicotina stomatitis, (B) Palatal changes in reverse smokers

13.3 times more site prediliction $(\mathrm{p}<0.001)$. Leukoplakia occurred predominantly in the buccal mucosa (15/37 cases) and commissural (14/37 cases) areas. Leukoplakia was 11.26 times significantly associated with commissural site $(\mathrm{p}<0.001)$.

In the sample size of 76, 40 patients (57.9\%) had smoking habit, $29(38.2 \%)$ had a habit of smokeless tobacco and the remaining $3(3.9 \%)$ patients had a combined habit of smoking and smokeless tobacco use (Fig. 5). In the smoking group, bidi was associated with more number of lesions when compared to cigarette smoking ( $\mathrm{p}<0.05$, Binomial probability). In the smokeless tobacco category, the most common habit associated with the lesions was the use of betel quid with tobacco (15/29). The other smokeless habits associated with the lesions were gutkha (7/29), khaini (2/29) and tobacco powder $(5 / 29)$.

Out of the total size, 29 lesions (38.2\%) occurred within 10 years duration of habit; 26 lesions (34.2\%) between 11 and 20 years while the remaining 21 lesions $(27.6 \%)$ occurred with the habit duration greater than 20 years. Fifty-four lesions (71.1\%) occurred with a frequency of 1 to 10 times of tobacco usage per day, fifteen lesions (19.7\%) occurred with the frequency ranging between 11 and 20 times per day and another seven lesions $(9.2 \%)$ with a frequency greater than 20 times per day.

All the cases were subjected to incisional biopsy and histopathological evaluation. Dysplastic changes were noticed in 50 cases $(65.8 \%)$ and the remaining 26 cases $(34.2 \%)$ did not show any signs of dysplasia. Out of the 50 cases which showed dysplastic changes, 27 (35.5\%) were mild (Fig. 6A), $17(22.4 \%)$ were moderate (Fig. 6B) and $6(7.9 \%)$ were of severe form (Fig. 6C) (Fig. 7). In the 50 cases with dysplasia, 28 cases were associated with the habit of smoking and the remaining 22 cases were associated with the habit of smokeless tobacco usage. Moderate to severe dysplasia is 1.83 times more likely to occur with smokeless tobacco usage when compared to smoked form $(\mathrm{p}=0.239)($ Graph 1).

Variation in the grades of dysplasia (moderate to severe) was evidently noticed in lesions associated with prolonged tobacco usage. Thus, the data obtained through this study revealed that patients with a duration of tobacco usage $>10$ years were 2.17 times more likely to have moderate-severe 

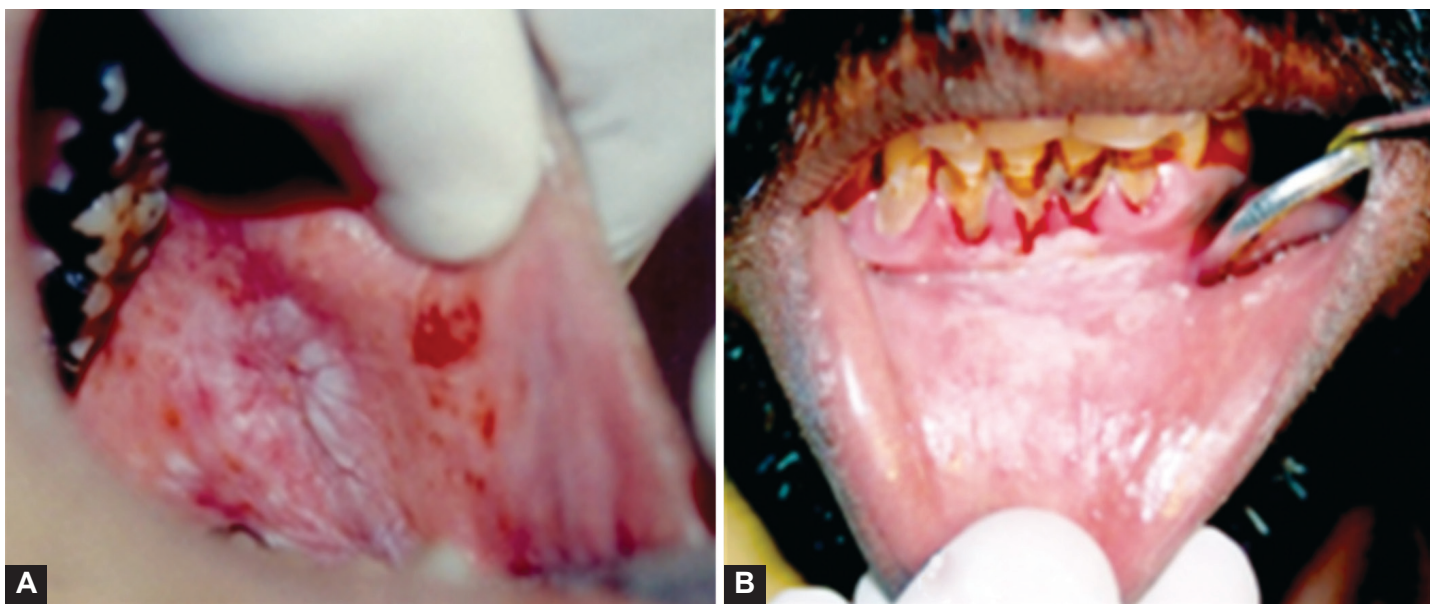

Figs 4A and B: Smokeless tobacco keratosis in the left buccal mucosa, (B) smokeless tobacco keratosis in the lower labial mucosa and vestibule

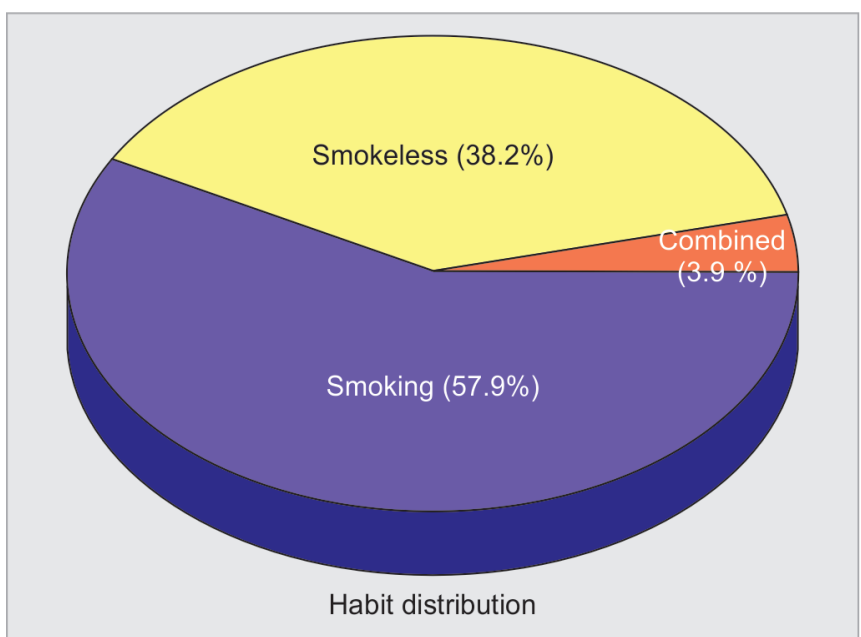

Fig. 5: Habit distribution

dysplasia $(\mathrm{p}=0.154)$. Moderate to severe dysplasia was 6.36 times more likely to occur in patients with tobacco usage at a frequency greater than 10 per day $(\mathrm{p}<0.01)$.

There was one case of erythroplakia which showed severe dysplasia. In the 28 cases of homogenous leukoplakia, twelve $(43 \%)$ showed no dysplasia; eleven (39\%) showed mild dysplasia; five (18\%) showed moderate dysplasia and none showed severe dysplasia. In the 9 cases of speckled leukoplakia, one (11.1\%) showed no dysplasia; three (33.3\%) showed mild dysplasia; two (22.2\%) showed moderate dysplasia and three (33.3\%) showed severe dysplasia. In the 9 cases of smoker's palate, four (44.4\%) showed no dysplasia; four (44.4\%) showed mild dysplasia; one (11.1\%) showed moderate dysplasia and none showed severe dysplasia. In the 29 cases of smokeless tobacco keratosis, nine (31\%) showed no dysplasia; nine (31.0\%) showed mild dysplasia; nine $(31.0 \%)$ showed moderate dysplasia and two (6.9\%) showed severe dysplasia. Patient's with speckled leukoplakia were 10.7 times significantly more likely to have severe dysplasia $(\mathrm{p}=0.020)($ Graph 2).

\section{DISCUSSION}

Tobacco is the leading preventable cause of premature death worldwide. It is a major independent risk factor for the development of oral and pharyngeal cancer. Malignant transformation is a multistep process that should be approached from the histological aspect also and not merely from the clinical standpoint. ${ }^{6}$ Thus, the present investigation was conducted for a clinicopathological evaluation of the various mucosal lesions associated with tobacco usage and to further assess any correlation between the duration and frequency of the habit, clinical appearance of the lesion and the grades of dysplasia, histopathologically.

With advancing age there is an increased tendency in the prevalence of precancerous lesions. The mean age of the total sample was 44.05 years (with a standard deviation of 11.85). This was consistent with the finding in similar studies conducted before.,

Smoking associated lesions were seen more in males $(96 \%)$ than in females $(4 \%)$. This study showed that smokeless tobacco keratosis is 24.11 times significantly more in females compared to males with $\mathrm{p}<0.00$, which led us to conclude that in this population group smokeless tobacco usage was predominant in females. This was in accordance with previous studies. ${ }^{9}$

In this study, homogeneous leukoplakia was found to be 3.12 times more prevalent than nonhomogeneous leukoplakia. These results are comparable to those of previous studies, 6.05 vs $1.40 \%, 1.26$ vs $0.21 \% .{ }^{10}$ Smokeless tobacco keratosis showed the highest occurrence in the buccal mucosa (25 in 29 cases) in this study. This could be attributed to the placement of the quid in the lower buccal sulcus. In the 37 cases with leukoplakia, 15 occurred in the buccal mucosa and 14 occurred in the commissural area. It was concluded that leukoplakia is 11.26 times significantly associated with commissural site $(\mathrm{p}<0.001)$. This was consistent with the findings in other studies. ${ }^{11,12}$ 


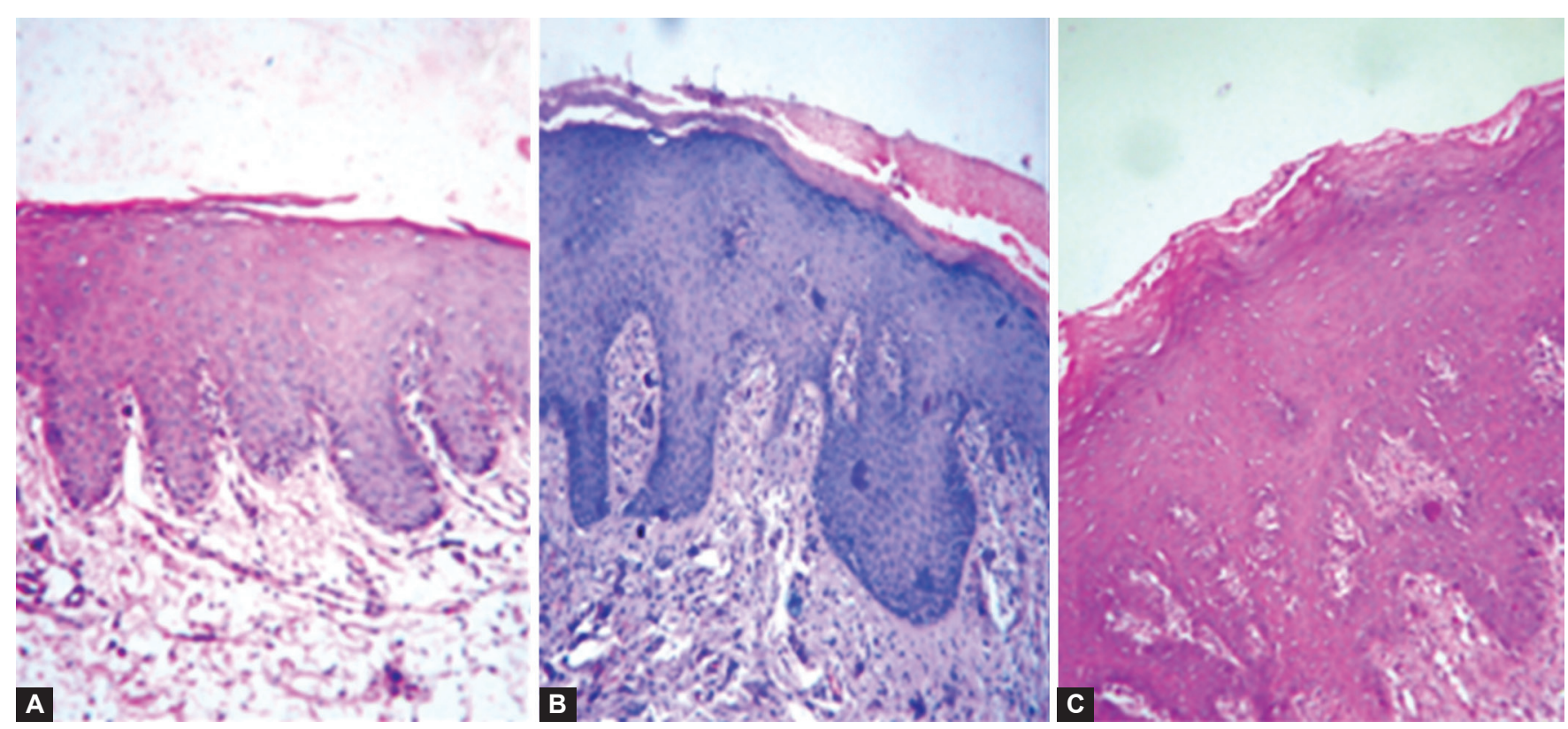

Figs 6 A to $C$ : Photomicrograph showing features of $(A)$ mild $(B)$ moderate and $(C)$ severe dysplasia

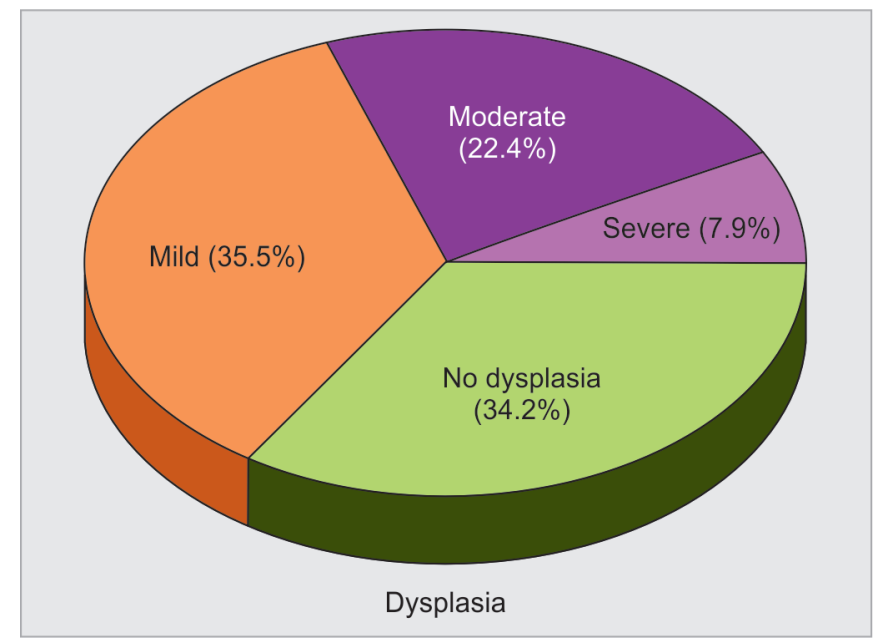

Fig. 7: Dysplasia

Tobacco is consumed in a variety of different ways, both in the smoked and smokeless forms. In this study $57.9 \%$ of patients with lesions had a smoking habit and $38.2 \%$ had habits associated with smokeless tobacco, and the remaining $3.9 \%$ patients had a combined habit of smoking and smokeless tobacco usage. In the smoking group, bidi was associated with more number of lesions when compared to cigarette smoking ( $p<0.05$, Binomial probability). This may be attributed to the type of tobacco used in manufacturing bidi as reported in a previous study wherein a meta-analysis of 12 case control studies reported an increased risk of bidi smoking for oral cancer. ${ }^{12}$

Analysis of data from this study revealed that lesions with smokeless tobacco usage were seen to occur within a short duration when compared to the smoking habit. This was in accordance with previous studies. ${ }^{13}$

The histopathological analysis of the lesions in this study showed a high percentage of dysplasias amounting to about

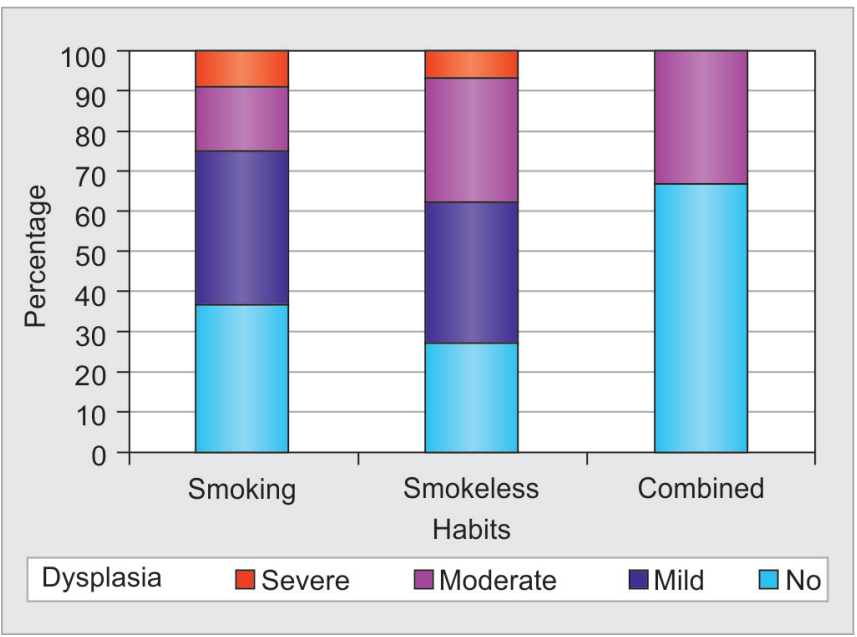

Graph 1: Association of habits with dysplasia

$65.8 \%$ ( 50 out of 76 cases). In the 50 cases with dysplasia, 29 cases were associated with the habit of smoking and 21 cases were associated with the habit of smokeless tobacco usage.

An interesting finding seen in this study was that moderate to severe dysplasia is 1.83 times more likely to occur with smokeless tobacco usage when compared to smoked form ( $p$ $=0.239)$. There was a study conducted in the Indian population in 1980, which showed that leukoplakias associated with chewing habits showed a higher rate of malignant transformation when compared to those associated with the habit of smoking. ${ }^{10}$ This was however in contradiction to results obtained in various studies done previously, which concluded that smokeless tobacco keratosis appears to show significant dysplastic changes less commonly than do other forms of clinical leukoplakia and erythroplakia. ${ }^{14}$ There were various studies on smokeless tobacco keratosis which showed a low percentage of dysplasia histopathologically ranging from 0 to $18 \%{ }^{13,15}$ This variation between previous studies and 


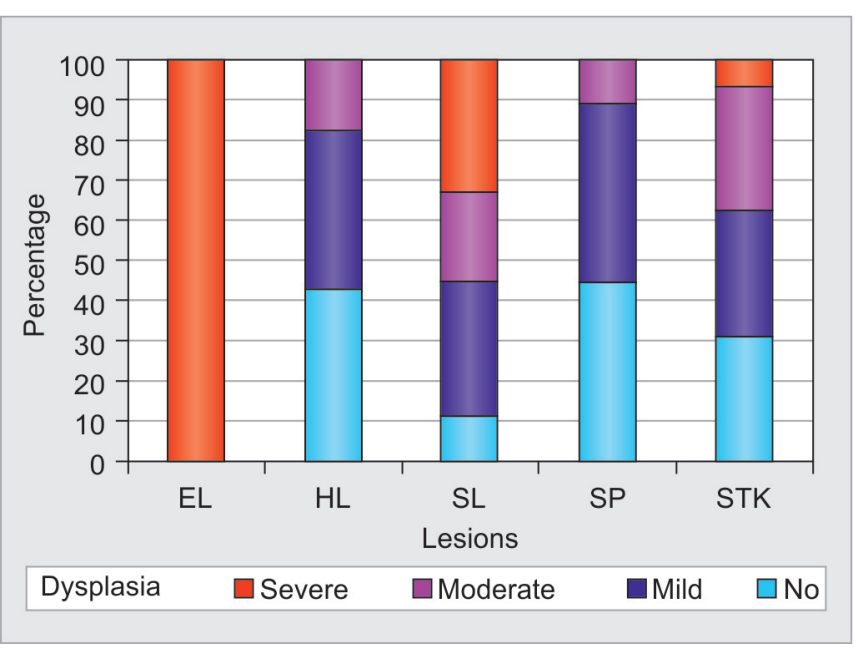

Graph 2: Association of lesions with dysplasia

the present study can be substantiated on the basis of type of smokeless tobacco used. The previous studies were mostly in the Western countries where smokeless tobacco usage is more in the form of snuff or loose tobacco powder, but in the present study conducted in a population of South India, betel quid with tobacco was the most prevalent habit. Quid is a substance or mixture of substances (in any manufactured or processed form) that is placed in the mouth, where it is sucked or actively chewed and thus remains in contact with the mucosa over an extended period. The major constituents of quid include Areca nut, slaked lime, betel leaf and tobacco. Recently in a study conducted in Kerala it was concluded that chewing betel quid without tobacco was an independent risk factor for oral precancers. ${ }^{16}$ This adds weight age to the fact that the constituents of betel quid will act in synergism with tobacco and enhance its carcinogenic potential.

A significant finding in this study was an increase in the moderate-severe dysplasia in the lesions associated with a tobacco habit of duration greater than 10 years and the frequency of intake greater than 10 times per day. Data obtained through this study revealed that patients with a duration of tobacco usage $>10$ years are 2.17 times more likely to have moderate-severe dysplasia with $\mathrm{p}=0.154$. Moderate to severe dysplasia is also 6.36 times significantly more likely to occur in patients with tobacco usage at a frequency greater than 10 per day $(\mathrm{p}<0.01)$. This finding is in accordance with other studies which also showed a similar dose-response relationship. ${ }^{12,17-19}$

In this study group of 76 patients with lesions, nine cases were speckled leukoplakia. In this nine cases, three (33.3\%) showed severe dysplasia histopathologically. Thus, it was concluded that patient's with speckled leukoplakia are 10.7 times significantly more likely to have severe dysplasia with $p=0.020$. Similar findings were seen in various other studies which revealed an increased risk for malignant transformation in cases of speckled leukoplakia. ${ }^{17,20}$ This could be attributed to the erosive component of the mucosa in this entity which leads to an enhanced action of the carcinogen due to a reduction in the mucosal barrier.

Significant numbers of dysplasia's especially the higher grades (moderate-severe) eventually transform into malignancy. ${ }^{5,21,22}$ At the opposite end of the spectrum, mild epithelial dysplasia so seldom eventuates in carcinoma (less than $5 \%$ of cases) and is so similar to reactive epithelial change, that few pathologists consider it a serious threat or recommend complete removal of the associated clinical lesion. ${ }^{22,23}$ It is always assumed, however, that the more severe dysplasia's must have begun life as less severe lesions and that a certain proportion of mild dysplasia's will progress to a more severe stage. It is justifiable in our present state of knowledge to consider in general terms that the degree of dysplasia is linked to the degree of probability of the development of malignancy. In any event it is a safe rule to advice the complete removal, wherever possible of all lesions showing evidence of dysplasia.

We do agree that advances in the utilization of new immune histochemical markers, molecular biology techniques and genomic investigations represent a considerable research field and an immense hope of improvement in early detection of malignancy and premalignancy. ${ }^{24}$ Application of these techniques is presently reserved to highly specialized laboratories and referral centers, and not available in the daily practice of most histopathologic laboratories. We hope that in the future a possible application of such techniques in routine work will contribute to early diagnosis and prompt treatment.

\section{CONCLUSION}

The key to better quality and length of survival is more effective detection of disease at an early stage/when the lesion is small. Today the future is relatively optimistic for patients whose disease is identified early. This highlights the important role of oral physicians in detecting oral mucosal lesions and screening their patients who fall into the known risk group for oral cancer and precancers, on a regular basis. The study also reaffirms the importance of public education, stressing the risk factors for oral cancer and precancers and the necessity of counseling patients with such lesions.

\section{REFERENCES}

1. Bouquot JE, Schroeder KL. The oral effects of tobacco abuse. J Amer Dent Inst Cont Educ 1992;43:3-17.

2. Mehta FS, Hamner JEI. Tobacco-related oral mucosal lesions and conditions in India, Basic Dental Research Unit, Tata Institute of Fundamental Research, Bombay, India 1993. 
3. Downer MC, Evans AW, Hallett H, Jullien JA, Speight PM, Zakrzewska JM. Evaluation of screening for oral cancer and precancer in a company headquarters. Community Dentistry and Oral Epidemiology 1995;23(2):84-88.

4. Cotran RS, Kumar V, Robbins SL. Pathologic basis of disease. 5th ed. Philadelphia. WB Saunders Company 1992.

5. Lumerman H, Freedman P, Kerpel S. Oral epithelial dysplasia and the development of invasive squamous cell carcinoma. Oral Surg Oral Med Oral Pathol Oral Radiol Endod 1995;79(3):321-329.

6. Kuffer R, Lombardi T. Premalignant lesions of the oral mucosa. A discussion about the place of oral intraepithelial neoplasia (OIN). Oral Oncol 2002;38:125-130.

7. Mehta FS, Pindborg JJ, Gupta PC, Daftary DK. Epidemiologic and histologic study of oral cancer and leukoplakia among 50915 villagers in India. Cancer 1969;24(4):832-849.

8. Mehta FS, Gupta PC, Daftary DK, Pindborg JJ, Choksi SK. An epidemiologic study of oral cancer and precancerous conditions among 101761 villagers in Maharashtra, India. Int J Cancer 1972;10(1):134-141.

9. Kaugars GE, Mehailescu WL, Gunsolley JC. Smokeless tobacco use and oral epithelial dysplasia. Cancer 1989;64(7):1527-1530.

10. Gupta PC, Mehta FS, Daftary DK, Pindborg JJ, Bhonsle RB, Jalnawalla PN, et al. Incidence rates of oral cancer and natural history of oral precancerous lesions in a 10-year follow-up-study of Indian villagers. Community Dent Oral Epidemiol 1980; 8(6):287-333

11. Gangadharan P, Paymaster JC. Leukoplakia: an epidemiologic study of 1504 cases observed at the Tata Memorial Hospital Bombay, India. Br J Cancer 1971;25(4):657-668.

12. Rahman M, Sakamoto J, Fukui T. Bidi smoking and oral cancer: a meta-analysis. Int J Cancer 2003;106(4):600-604.

13. Vigneswaran N, Dent M, Tilashalski K, Rodu B, Cole P. Tobacco use and cancer. Oral Surg Oral Med Oral Pathol Oral Radiol Endod 1995;80(2):178-182.

14. Mincer HH, Coleman SA, Hopkins KP. Observations on the clinical characteristics of oral lesions showing histologic epithelial dysplasia. Oral Surg Oral Med Oral Path 1972;33(3): 389-399.

15. Kaugars GE, Riley WT, Brandt RB, Burns JC, Svirsky JA. The prevalence of oral lesions in smokeless tobacco users and an evaluation of risk factors. Cancer 1992;70(11):2579-2585.

16. Jacob BJ, Straif K, Thomas G, Ramadas K, Mathew B, Zhang ZF, Sankaranarayanan R, Hashibe M. Betel quid without tobacco as a risk factor for oral precancers. Oral Oncol 2004;40(7):697-704.

17. Jaber MA, Porter SR, Gilthorpe MS, Bedi R, Scully C. Risk factors for oral epithelial dysplasia: the role of smoking and alcohol. Oral Oncol 1999;35(2):151-156.

18. Jaber MA, Porter SR, Scully C, Gilthorpe MS, Bedi R. The role of alcohol in nonsmokers and tobacco in nondrinkers in the aetiology of oral epithelial dysplasia. Int J Cancer 1998;77(3): 333-336.

19. Idris AM, Warnakulasuriya KA, Ibrahim YE, Nielsen R, Cooper D, Johnson NW. Toombak-associated oral mucosal lesions in Sudanese show a low prevalence of epithelial dysplasia. J Oral Pathol Med 1996;25(5):239-244.

20. Dietrich T, Reichart PA, Scheifele C. Clinical risk factors of oral leukoplakia in a representative sample of the US population. Oral Oncol 2004;40(2):158-163.

21. Schepman KP, Van der Meij EH, Smeele LE, Van der Waal I. Malignant transformation of oral leukoplakia: a follow-up study of a hospital-based population of 166 patients with oral leukoplakia from Netherlands. Oral Oncology 1998;34(4): 270-275.

22. Scully C, Sudbo J, Speight PM. Progress in determining the malignant potential of oral lesions. J Oral Pathol Med 2003; 32(5):251-256.

23. Jaber MA, Porter SR, Speight P, Eveson JW, Scully C. Oral epithelial dysplasia: clinical characteristics of western European residents. Oral Oncol 2003;39(6):589-596.

24. Sudbo J, Reith A. 'When is an oral leukoplakia premalignant?' Oral Oncology 2002;38(8):813-814. 